\title{
HEAT TRANSFER AUGMENTATION IN DIFFERENT GEOMETRIES OF DIMPLED SURFACE UNDER NATURAL CONVECTION AN EXPERIMENTAL APPROACH
}

\author{
M.A.Dafedar ${ }^{1}$, Najeem $\mathbf{M}^{2}$, Fakruddin $\mathbf{Y}^{\mathbf{3}}$, Chirag $\mathbf{P}^{\mathbf{4}}$ \\ ${ }^{1}$ Assistant Prof, ${ }^{2,3,4} U G$ Scholar, Department of Mechanical Engineering, \\ SECAB Institute of engineering and Technology, Bijapur, India \\ msadafedar@gmail.com,najeem7411@gmail.com, fjy8@rediffmail.com,chiragpatel007007@yahoo.co.in
}

\begin{abstract}
The prime objective of present work is to study experimentally the heat transfer augmentation through various geometries of dimpled surfaces in longitudinal and lateral directions. In this paper horizontal rectangular plates of copper and aluminum with different dimpled geometries (like square, circular and triangular) for in-line arrangements were studied in natural convection with steady laminar external flow condition. The various parameters considered for study are Nusselt number, heat transfer coefficient and heat transfer rate for a constant Prandtl number (0.7) and Grashof number $\left(10^{4}-10^{7}\right)$.It has been found that the heat transfer coefficient and heat transfer rate increases for various dimpled surfaces as compared to plane surface. It has been also found that the heat transfer coefficient and heat transfer rate increases along longitudinal direction as compared to lateral direction. And it is seen that heat transfer rate is maximum for triangular shape dimple when the apex of triangle is faced towards inlet of air flow Finally it is concluded that heat transfer enhancement takes place along the dimpled surface.
\end{abstract}

Index Terms: Dimpled plates, Natural Convection, Heat transfer augmentation.

****

\section{INTRODUCTION}

Heat transfer Enhancement techniques are commonly used in areas such as process industries, heating and cooling in evaporators, thermal power plants, air-conditioning equipment, refrigerators, radiators for space vehicles, automobiles, Electronic devices, Chemical process etc. Over the past couple of years the focus on using dimples on surface for intensifying the heat transfer has been documented by many researchers. A variety of experimental, analytical and Numerical research work has been carried out on augmentation of heat transfer. In 1971 Kuethe [1] was first to suggest the use of dimple on surface for heat transfer enhancement, according to him the dimples are expected to promote vortex generation which results in heat transfer enhancement. V.N Afnasyev [2] have conducted experimental study on surfaces shaped by systems of spherical cavities and they found that heat transfer was increased by $150 \%$ as compared to plane surface.Nikolai Kornev[3] have studied vortex structure and heat transfer enhancement in turbulent flow over staggered dimple array in narrow channel by using Large Eddy Simulation. Mahmood and Ligrani [4] have analyzed experimentally the influence of dimple aspect ratio, temperature ratio, Reynolds Number, and flow structure in dimple channel. Z Wang [5] carried out Numerical simulation of laminar channel flow over dimple surface and identified a symmetric 3D horseshoe vortex inside a single dimple.
S.L.Borse and I.H Patel [6] carried out an experimental study on effect of dimples on heat transfer over flat surface under forced convection. They reported the use of dimples on surface results in heat transfer enhancement with lesser pressure drop and also stated that heat transfer enhancement is more effective in staggered arrangement compared to in line arrangement. Moon [7] studied the channel height effect on heat transfer over the dimpled surfaces. Heat transfer coefficient and friction factors were computationally investing-ated in rectangular channels, which had dimples on one wall. Although abundant studies have been accomplished in this field, but there are not many Papers that have investigated the effect of different dimple geometries on heat transfer rate under laminar external flow Natural convection.

\section{EXPERIMENTAL PROCEDURE}

The block diagram shown below is experimental set up for present study. The set up mainly consist of heater with capacity of 200 watts, Dimmer stat, Digital temperature, voltmeter, and ammeter with $\mathrm{J}$ type thermocouple. The test plates were placed on heater in a rectangular duct open to atmosphere at inlet and outlet. A constant heat is supplied through dimmer stat to heater. Air flows parallel to the dimpled test surface. The plate heater is fixed at the bottom of the test plate, and was connected to power socket through dimmer stat. Dimmer stat readings were varied to give the 
required heat input to the test plate. Only top dimpled surface of the test plate was exposed to the air stream from which the convective heat transfer to the air stream takes place. After reaching a steady state the inlet and outlet temperature of air with surface temperature of plate and dimples were measured with the help of thermocouple.

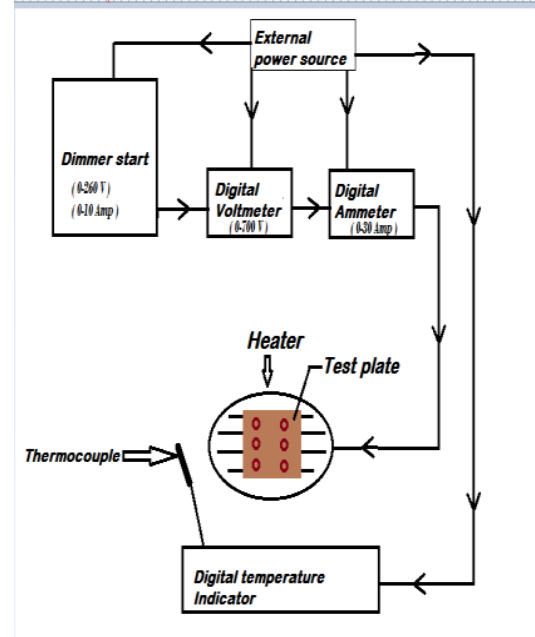

Block Diagram of Epperimental setup

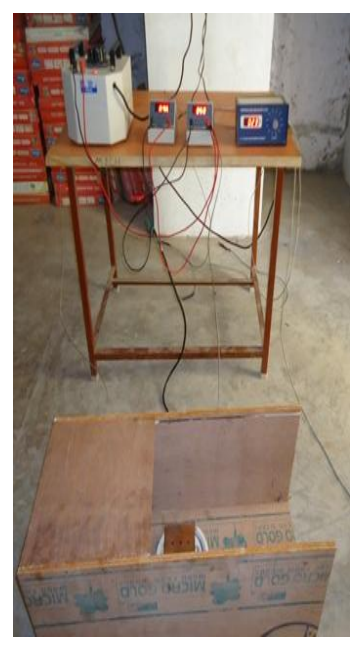

Fig-1b
Fig-1(a \& b): Experimental Set Up

\subsection{TEST PLATES}

Triangular dimple

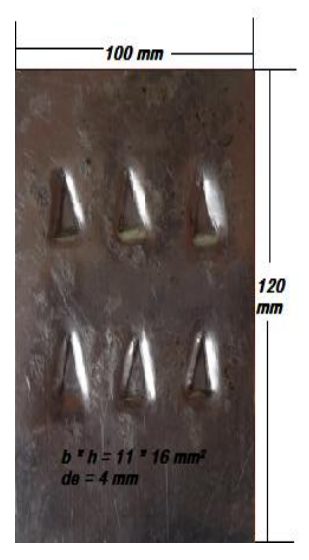

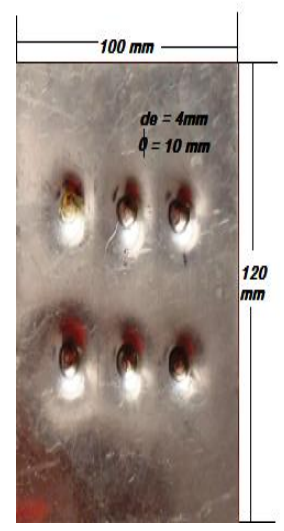

Circular Dimple

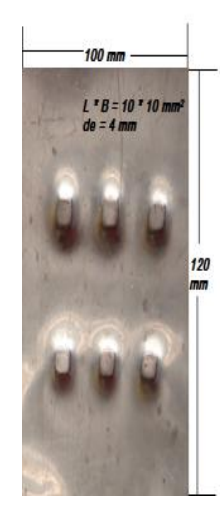

Square Dimple
Fig-2: Different dimple geometries

The three different geometries were used for present study i.e. Square, Triangular and Circular.Above Figure shows different dimple geometries on rectangular plate of size $100 \mathrm{~mm} \mathrm{x}$ $120 \mathrm{~mm}$. The size of square dimple is $10 \mathrm{mmX} 10 \mathrm{~mm}$, triangular dimple is $11 \mathrm{mmX} 16 \mathrm{~mm}$, circular dimple of dia $10 \mathrm{~mm}$ were used. The depth of all the dimples is kept constant and six dimples on each plate with inline arrangement were used.

\subsection{DATA REDUCTION}

The study is carried out under natural convection for external laminar flow condition. Steady state value of the plate and air temperatures in the channel, at various locations for a given heat flux is used to determine the values of performance parameters.

A) Grash of number

$\mathrm{Gr}=\mathrm{g} * \beta * \mathrm{~L} 3 * \Delta \mathrm{T} / \mathrm{v} 2$

Where

$\mathrm{g}=$ Acceleration due to gravity $9.81 \mathrm{~m} / \mathrm{s} 2$

$\mathrm{L}=$ Length of the Plate in meters

$v=$ Kinematic Viscosity at film temp in $\mathrm{m} 2 / \mathrm{s}$.

$\beta=$ Coefficient of volumetric expansion in $\mathrm{K}-1$

$\Delta \mathrm{T}=$ Temperature difference in $\mathrm{K}$

B) Nusselt number

$\mathrm{Nu}=0.54(\mathrm{Gr} * \operatorname{Pr}) 1 / 4$

Pr is Prandtl number

C) Average heat transfer coefficient ha= $\mathrm{Nu} *$ Kair/ L

Kair= Thermal conductivity of air at film temperature in $\mathrm{W} / \mathrm{m}^{\circ} \mathrm{c}$

D) Rate of heat transfer

$\mathrm{Qa}=$ ha A (Ts-Tळ)

$\mathrm{Qa}=$ Average heat transfer rate in Watts

\section{RESULTS AND DISCUSSION}

From figure 3 , it has been found that heat transfer coefficient for plane surface is more in copper plate as compared to aluminum plate for same surface area. And it is also found that the heat transfer coefficient is high for triangular dimpled surface facing apex towards flow in copper plate compared to aluminum plate. But for square and triangular (base facing towards flow) dimpled surface the heat transfer coefficient is more in aluminum plate compared to copper plate

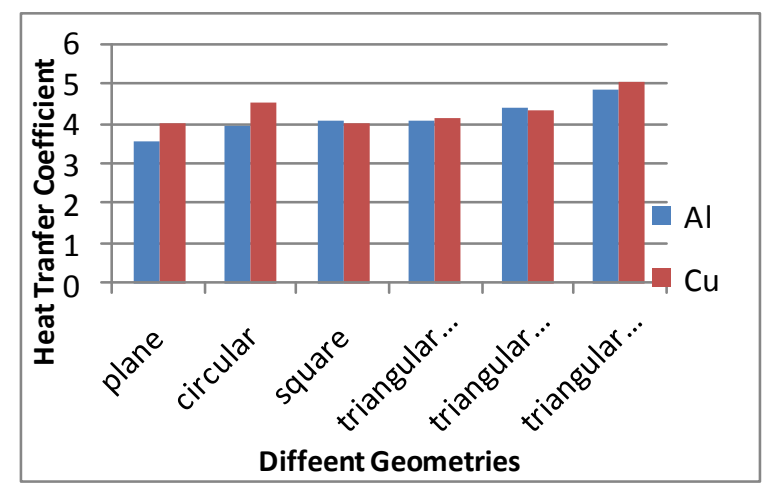

Fig-3: Variation of heat transfer coefficient With different geometries 
Table-1: Results for Copper Plate

\begin{tabular}{|l|l|l|l|l|l|l|}
\hline & plane & circular & Square & $\operatorname{Tri}(\mathrm{H})$ & $\operatorname{Tri}(\mathrm{B})$ & $\operatorname{Tri}(\mathrm{A})$ \\
\hline $\mathrm{h}_{\mathrm{a}}$ & 3.98 & 4.51 & 4.024 & 4.119 & 4.313 & 5.02 \\
\hline $\mathrm{N}_{\mathrm{u}}$ & 17.82 & 19.90 & 17.78 & 18.18 & 15.93 & 18.47 \\
\hline $\mathrm{Q}_{\mathrm{a}}$ & 0.375 & 0.778 & 0.429 & 0.444 & 0.508 & 1.08 \\
\hline
\end{tabular}

From figure 4, it has been found that the Nusselt number for copper plate with plane surface, circular and triangular (apex facing towards flow) dimpled surface is found to be more compare to aluminum plate. It is also found that the Nusselt number for aluminum plate with square and triangular (base and hypotenuse facing towards flow) dimpled surface is more compared to copper plate.

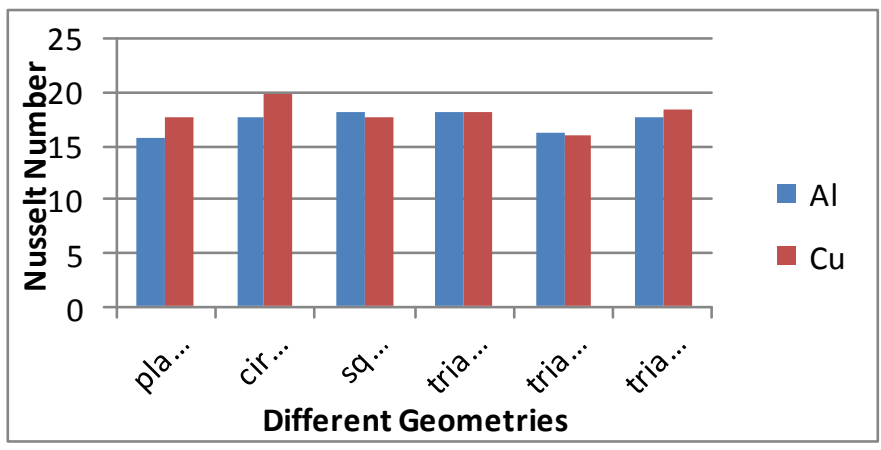

Fig-4: Variation of Nusselt number with Different geometries

Table-2: Results for Aluminum Plate

\begin{tabular}{|l|l|l|l|l|l|l|}
\hline & plane & circular & Square & $\operatorname{Tri}(\mathrm{H})$ & $\operatorname{Tri}(\mathrm{B})$ & $\operatorname{Tri}(\mathrm{A})$ \\
\hline ha & 3.557 & 3.964 & 4.094 & 4.075 & 4.413 & 4.81 \\
\hline $\mathrm{Nu}$ & 15.84 & 17.60 & 18.31 & 18.32 & 16.252 & 17.64 \\
\hline Qa & 0.213 & 0.409 & 0.489 & 0.454 & 0.564 & 0.874 \\
\hline
\end{tabular}

From figure 5, it has been found that the heat transfer rate for copper plate with plane surface, circular and triangular (apex facing towards flow) dimpled surface is found to be more compare to aluminum plate. It is also found that the heat transfer rate for aluminum plate with square and triangular (base and -hypotenuse facing towards flow) dimpled surface is more compared to copper plate.

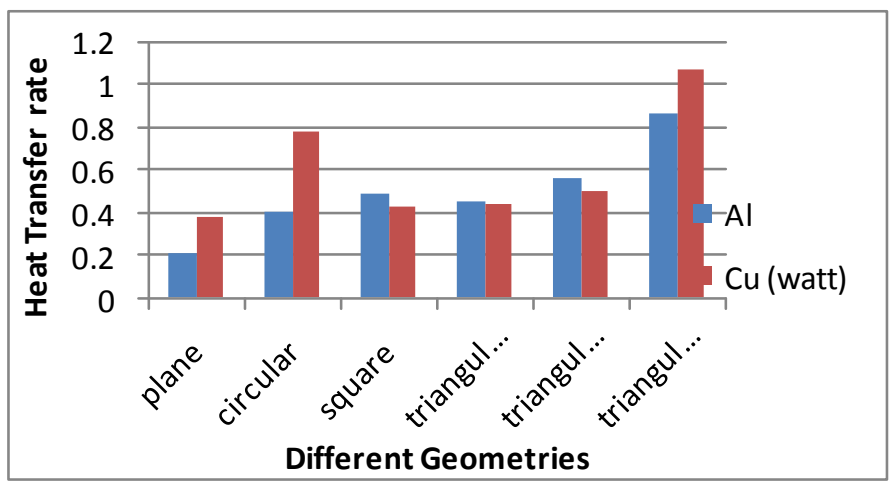

Fig-5: Variation of heat transfer rate with Different geometries

\section{CONCLUSIONS}

From the present study the following conclusion were made:

- It is found that the heat transfer rate is more for different dimpled geometry compare to plane plates (both for aluminum and copper).

- It is concluded that the maximum heat transfer rate will takes place in triangular dimpled surface with apex facing towards inlet of air flow in both plates.

- It is also concluded that the minimum heat transfer rate takes place in square dimpled surface of copper plate compared to other geometries.

- It is found that the Nusselt number is high for circular dimpled surface in copper plate and triangular dimpled surface (hypotenuse facing towards air flow) in aluminum plate.

- The Nusselt number is low both in aluminum and copper plate for triangular dimpled surface with base facing towards air flow.

- The heat transfer coefficient is maximum for both aluminum and copper plate in triangular dimpled surface with apex facing towards air flow.

\section{ACKNOWELEGEMENTS}

We would like to thank our principal and management for their support and help towards this work.

\section{REFERENCES}

[1] Kuethe A. M., (1971). Boundary Layer Control of Flow Separation and Heat Exchange, US. Patent No. 3,578,264.

[2] V.N., Afanasyev, Ya.P., Chudnovsky, A.I., Leontiev, P.S., Roganov, Turbulent flow friction and heat transfer characteristics for spherical cavities on a flat plate, Experimental Thermal and Fluid Science 7 (1) (1993) 1-8.

[3] Nikolai Kornev. Flow structures and heat transfer on dimpled surfaces (http://www.tsfp7.org/papers/2B4P.pdf).

[4] Mahmood, G. I., Ligrani, P. M., "Heat Transfer in a dimpled channel: combined influences of aspect ratio, 
temperatur6e ratio, Reynolds number and flow structure", International Journal heat Mass Transfer 45, 2011-2020, 2002. [5] Z., Wang, K.Z., Yeo, B.C., Khoo, Numerical simulation of laminar channel flow over dimpled surface, AIAA Paper No. AIAA 2003-3964.

[6]. Dr.S.L.Borse and I.H. Patel," Experimental investigation of heat transfer enhancement over the dimpled surface", Iftikarahamad H. Patel et al. / International Journal of Engineering Science and Technology (IJEST) ISSN: 09755462 Vol.4 No.8 August 2012 (PP no 3666-3672).

[7] Moon H.K., T. O'Connell, Glezer B., "Channel Height Effect on Heat Transfer and Friction in a Dimpled Passage", ASME J. Gas Turbine and Power, 122, April 2000, Pp.307313.

\section{BIOGRAPHIES}

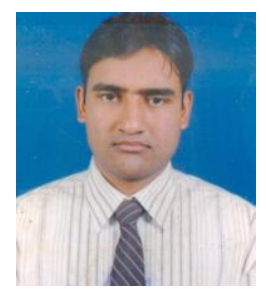

M. A. Dafedar has done his M.Tech in Production Technology from Basveshwar Engineering college Bagalkot in 2006. He is working as an Assistant Prof and HOD in mechanical engineering Department of SECAB IET BIJAPUR. He has got an experience of 8 years in teaching and 1 year industry. He has guided many UG Projects. Currently he is pursuing his PhD (Heat Transfer) from Visvesvaraya Technological University Belgaum. His area of interest is Fluid mechanics, Heat transfer, Operations research and Machine drawing.

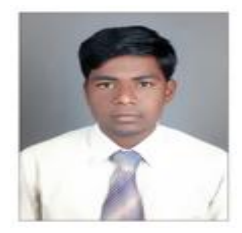

Najeem Makandar is pursuing his B.E. in Mechanical Engineering from Secab institute of engineering and technology Bijapur his subject of interest is Heat Transfer, Thermodynamics and Vibration.

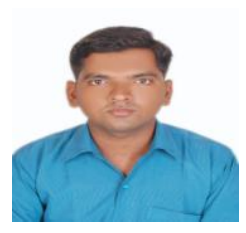

Fakruddin Yendigiri is pursuing his B.E. in Mechanical Engineering from Secab institute of engineering and technology Bijapur his subject of interest is Heat Transfer, Thermodynamics and Fluid Mechanics.

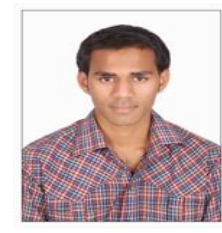

Chirag Patel is pursuing his B.E. in Mechanical Engineering from Secab institute of engineering and technology Bijapur his subject of interest is Heat Transfer, Thermodynamics and Control engineering. 